\title{
Optimization of Layered Space Time Codes for MIMO Underwater Sensor Communication
}

\author{
Chang-Uk Baek ${ }^{1, a}$, Ji-Won Jung ${ }^{2, b}$ \\ ${ }^{1,2}$ Department of Radio Communication Engineering, Korea Maritime and Ocean University, Busan \\ 606-791, Korea \\ a cuback@kmou.ac.kr, bjwjung@kmou.ac.kr \\ Corresponding Author : Ji-Won Jung
}

Keywords: Space time trellis codes, Turbo equalization, Single-Output-Single-Input, Multiple-Output-Multiple-Input, BCJR, Underwater communication channel

\begin{abstract}
In this paper, we expand iterative turbo equalizer techniques for single-input-single-output (SISO) system to multiple-input-multiple-output (MIMO) system in order to increase data rates for multipath channel environment such as underwater communication channel. In MIMO system, space time trellis codes (STTC) was employed as an inner code. In receiver side, BCJR algorithm is used for STTC decoding in order to improve BER performance by increasing iterations. Based on experimental channel response, we confirmed that the performance is the better as iteration number is increased.
\end{abstract}

\section{Introduction}

The excessive multipath encountered in underwater sensor communication (USC) channel is creating inter symbol interference (ISI), which is limiting factor to achieve a high data rate and bit error rate (BER) performance. Among the various ways to cope with ISI, one well known is iterative equalizer which constitutes an outer loop in the receiver. An inner loop consists of iterative decoder. The assembly utilizes error correcting power of the iterative codes to get an efficient equalizer [1,2]. Based on iterative turbo equalization technique for single-input-single-output (SISO) channel, this paper expands it to the multiple-input-multiple-output (MIMO) channel for increasing data rates and capacity gains $[3,4]$.

MIMO technique is being studied in underwater communications because of increasing the data rates. MIMO communication systems employ multiple sensors at the transmitter and receiver sides. They can yield significantly increased data rates and improved link reliability without additional bandwidth [5]. In MIMO system, space time code (STC) was employed as an inner code. As the same as SISO system, the inner codes are turbo codes. In receiver side, BCJR algorithm is used for STC decoding in order to improve BER performance by increasing iterations. This paper gives optimal structure for MIMO underwater system by simulation using the some sea trials conducted in the East-sea.

\section{MIMO System for Underwater Communication}

System model. Consider an N x M MIMO communication system equipped with $\mathrm{N}$ transmit transducers and $\mathrm{M}$ receive transducers. The individual data streams of each transmitter are symbol aligned and are sent simultaneously. The data streams of each transmitter consist of successive data packages. The data packages start with a training sequence which is followed by the payload sequence. Fig. 1 shows the proposed N x M MIMO system structure based on turbo equalization. The source bits are encoded by STTC encoder and interleaved, then mapping to QPSK symbols, After the signals have been received by the receive array, the processing consists of estimating the channel impulse response in training or decision mode and detecting the symbols by using the estimated channel impulse response. For increase data rate and diversity gain according to using MIMO 
estimation and symbol detection has been done, significant performance improvement iterative turbo equalization BCJR(bahl, Cocke, Jelinek, Raviv) algorithm [6] for STTC decoding, de-interleaving and turbo decoding are performed. The baseband equivalent signal received at the $\mathrm{m}^{\text {th }}$ hydrophone can be expressed in the discrete-time domain form as

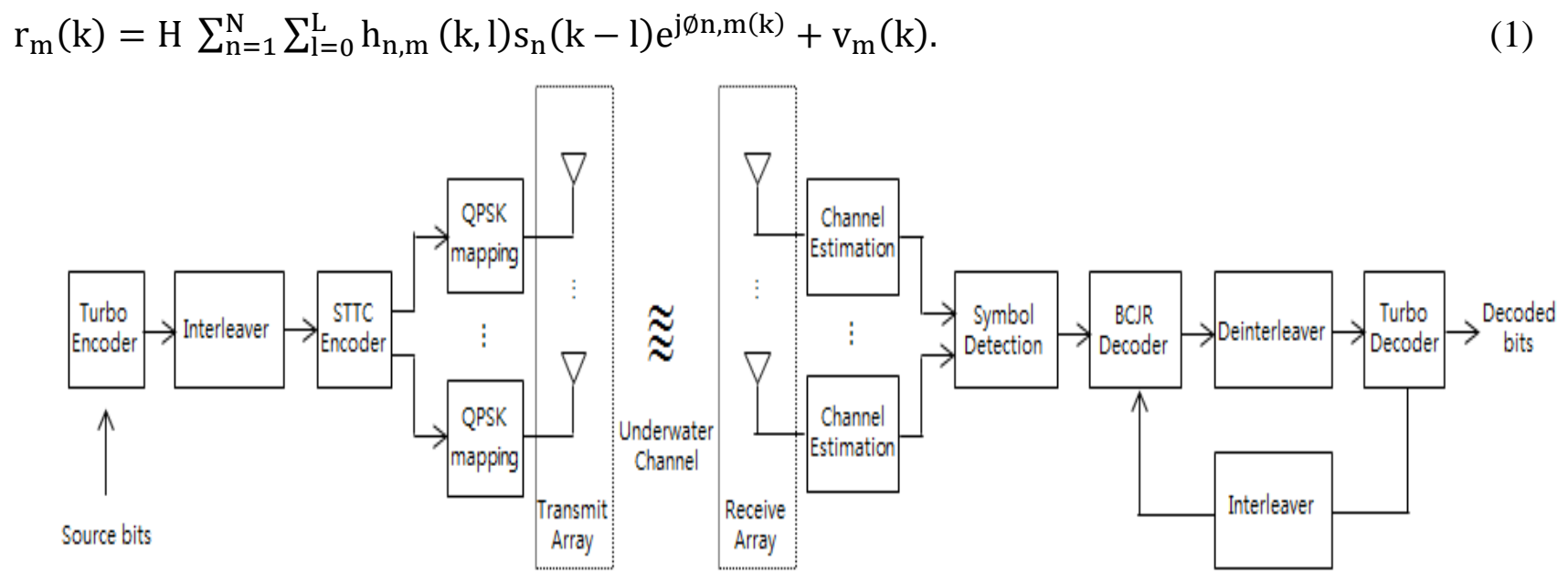

Fig. 1 N x M MIMO system structure based on turbo equalization

where $\mathrm{k}$ is the time index, $\mathrm{s}_{\mathrm{n}}(\mathrm{k})$ is the transmitted data symbol or training symbol from $\mathrm{n}^{\text {th }}$ transducer, and $h_{n, m}(k, l)$ is the channel impulse response of the frequency-selective, time varying fading channel with length $\mathrm{L}+1$ between $\mathrm{n}^{\text {th }}$ transducer and $\mathrm{m}^{\text {th }}$ hydrophone. $\mathrm{v}_{\mathrm{m}}(\mathrm{k})$ means an additive white Gaussian noise.

At the receiver, we resort to powerful turbo equalization algorithms that iteratively exchange probabilistic information between inner decoder and outer decoder, thereby reducing the error rates significantly. The symbols of outer decoder are then subtracted from the input and interleaved. The interleaved symbols are canceled a posteriori from the proceeding received symbol. Interleaving helps receiver convergence. To confirm the performance improvement of the iterative turbo equalization for MIMO system, the simulation was conducted. Underwater communication is difficult to maintain the reliability because it is affected by temperature, depth and geometry.

Optimum space time codes for MIMO underwater channel. In MIMO turbo equalization, two codes are concatenated in the serial fashion. The inner codes are turbo codes with 16 states, and outer codes are space time codes. Normally, candidate of outer codes are space-time block codes (STBCs) and space time trellis codes (STTCs). Representative method of STBCs is V-BLAST (Vertical-Bell Labs layered Space-Time) [7][8]. This system is obtained diversity or spatial multiplexing effect. The MLD is optimal and fully exploits the available diversity. However, STBCs for MIMO turbo equalization can't obtain coding gain even if increasing number of iteration. This is the reason that the outputs of STBCs are not soft type symbols. The types of input symbols and output symbols must be soft symbols in order to improve performance by increasing number of iterations.

BCJR algorithm for STTC decoder. Generally, the decoding method of the convolutional code is a Viterbi decoding by using a hard decision value. However, to improve performance through iteration with DFE, soft decision value is required. Therefore, we used BCJR algorithm with soft decision value. BCJR algorithm is a well-known maximum a posteriori probability decoding algorithm which has been proposed earlier for point to point communication applications.

A set of triples (previous state of $s^{\prime}$, channel output of $r_{j}$, next state of $s$ ) uniquely defines a finite state machine on which the BCJR operates. It has 16 states, and each state is given by a different 4-bit pattern. The states in vertical columns represent all possible states that the channel can take at a given time instant, while the labeled edges represent possible transitions. The log-likelihood ratio (LLR) of input bit of $s_{j}(j=1,2, \ldots, n)$, is calculated as 


$$
\begin{array}{r}
\mathrm{L}\left(\mathrm{u}_{\mathrm{j}}\right)=\max ^{*}\left[\alpha_{j-1}\left(\mathrm{~s}^{\prime}\right)+\gamma_{\mathrm{j}}\left(\mathrm{s}^{\prime}, \mathrm{s}\right)+\beta_{\mathrm{j}}(\mathrm{s})\right]\left(\mathrm{s}^{\prime}, \mathrm{s}\right): \mathrm{u}_{\mathrm{j}}=0 \\
-\max ^{*}\left[\alpha_{j-1}\left(\mathrm{~s}^{\prime}\right)+\gamma_{\mathrm{j}}\left(\mathrm{s}^{\prime}, \mathrm{s}\right)+\beta_{\mathrm{j}}(\mathrm{s})\right]\left(\mathrm{s}^{\prime}, \mathrm{s}\right): \mathrm{u}_{\mathrm{j}}=1
\end{array}
$$

$\alpha_{j}(s), \beta_{j}(s)$ and $\gamma_{\mathrm{j}}\left(\mathrm{s}^{\prime}, \mathrm{s}\right)$ means forward metric, backward metric and branch metric.

Where, max $^{*}$-operator is defined as

$$
\max ^{*}(x, y)=\max (x, y)+\log \left(1+e^{|x-y|}\right)
$$

Channel estimation and equalizer. The channel estimation problem is to estimating $h_{m}$ from the measurement $r_{m}$ and known $s_{j}$. Existing techniques for sparse channel estimation can be categorized roughly into two types [9]. Among the explicit sparse channel estimation techniques are the $L_{p}$-norm regularized method and greedy algorithms such as the matching pursuit algorithm. In this paper, we use the sparse channel estimation with dominant tap detection by using L1-norm minimization. L1-norm minimization is used for method of estimate $\mathrm{h}_{\mathrm{m}}$ as following equation.

$$
h_{m}=\operatorname{argmin}\|S\|_{1} \text { subject to }\left|r_{m}-S h_{m}\right|<\epsilon .
$$

Then the values of the channel responses are clipped as following.

$$
\mathrm{h}_{\mathrm{n}, \mathrm{m}}(\mathrm{l})= \begin{cases}\text { non }- \text { zero } & \text { if } \mathrm{h}_{\mathrm{n}, \mathrm{m}}(\mathrm{l})>\text { threshold } \\ \text { zero } & \text {, else }\end{cases}
$$

This dominant tap detection is performed that if the estimated value $h_{n, m}(l)$ is bigger than particular value, it would have non-zero value and the residuals will have zero value. The value $\hat{h}_{m}$ is the channel information corresponding to the estimated nonzero tap. In this paper, we use the zero forcing equalizer and DFE equalizer for ISI cancellation.

\section{Simulation Results and Performance Evaluation}

MIMO Underwater Channel Modeling. To confirm the performance improvement of the iterative turbo equalization for MIMO system, the simulation was conducted. Underwater communication is difficult to maintain the reliability because it is affected by temperature, depth and geometry. The channels for simulation were generated by Bellhop model, and the sound speed profile that was measured via sea trials was used. We considered 2 × 2 MIMO system. Fig. 2 (a) $\sim$ (d) are channel impulse responses between the transmitters and receivers. As expected, numerous reflections can be observed due to surface and bottom. The range between sources and receivers is $1[\mathrm{~km}]$, and the water depth is $200[\mathrm{~m}]$. The transmitters' and receivers' depth are $110[\mathrm{~m}]$ and $117[\mathrm{~m}]$. The carrier frequency is $12[\mathrm{kHz}]$, and the sampling frequency is $192[\mathrm{kHz}]$. The preamble signal was modulated by BPSK with data rate of 0.5 [kbps]. Source data were modulated by QPSK with data rate of 2 [kbps]. The underwater channel characterization for multipath environment is measured using 2000 [symbols] of PN code symbols.

The channels for simulation were generated by Bellhop model, and the sound speed profile that was measured via sea trials was used. We considered $2 \times 2$ MIMO system. The range between sources and receivers is $1[\mathrm{~km}]$, and the water depth is $200[\mathrm{~m}]$. The transmitters' and receivers' depth are $110[\mathrm{~m}]$ and $117[\mathrm{~m}]$. The carrier frequency is $12[\mathrm{kHz}]$, and the sampling frequency is $192[\mathrm{kHz}]$. The preamble signal was modulated by BPSK with data rate of 0.5 [kbps]. Source data were modulated by QPSK with data rate of 2 [kbps]. The underwater channel characterization for multipath environment is measured using 2000 [symbols] of PN code symbols. Table 1 listed simulation parameters for deciding optimal transceiver structure in MIMO underwater communication 

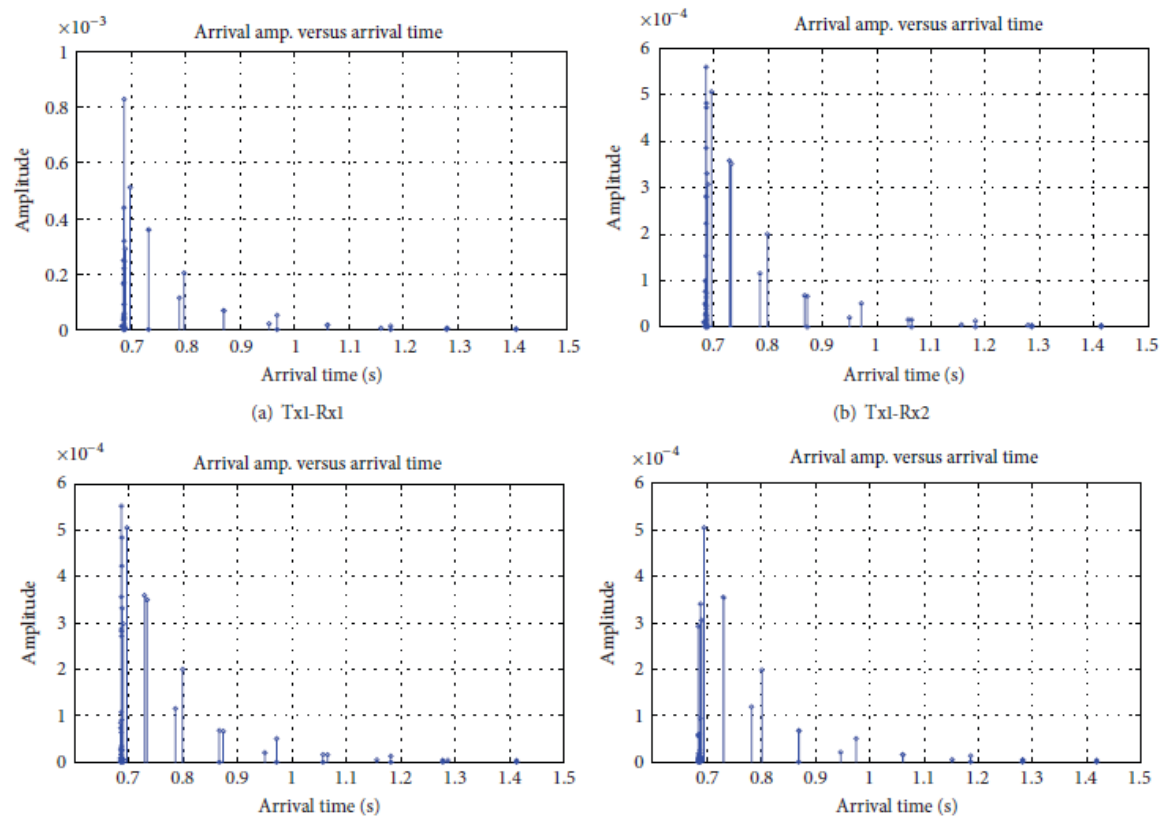

Fig. 2 Channel impulse responses for measured 2x2 MIMO system

Table 1. SIMULATION PARAMETER

\begin{tabular}{ll}
\hline Method & Parameters \\
Inner codes & Turbo codes \\
Outer codes & STBC, STTC \\
Channel equalizer & ZF, DFE \\
Modulation & QPSK \\
Data rate & $2[\mathrm{kbps}]$ \\
Carrier/sampling frequency & $16 / 192[\mathrm{kHz}]$ \\
Distance & $1[\mathrm{~km}]$ \\
\hline
\end{tabular}

Performance of outer codes. Fig. 3 shows BER curves for STBC and STTC for underwater channel. We confirmed the performance of STTC is better than that of STBC types coding techniques. This is the reason the trellis type coding schemes are more optimal for iterative decoder. We also know that STBC for MIMO turbo equalization can't obtain coding gain even if increasing number of iteration because the outputs of STBC are not soft type symbols.

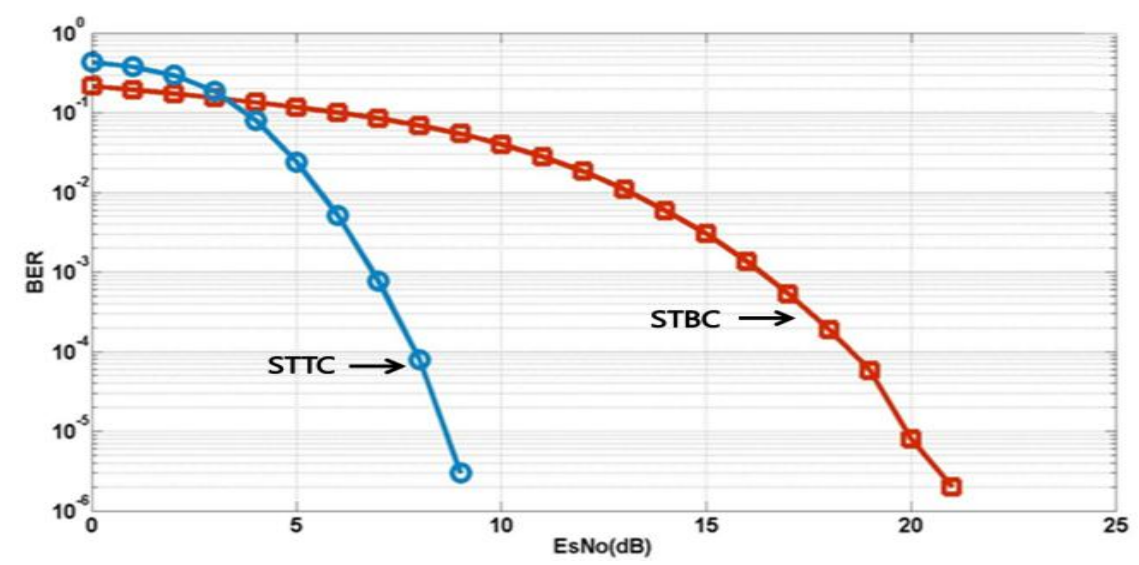

Fig .3 The performance comparison of STTC and STBC

Performance of channel equalization types. To mitigate ISI in underwater channel, two kinds of channel equalizations are used in this paper. One well proven method is zero forcing method which 
fixes tap coefficients using the inverse channel metrics. The structure is relative simple, however tap coefficients are not changed during payload period. If channel states are change during payload period, the performance would be degraded. Another one is DFE method. During the training mode, tap coefficients are fixed using the preamble data. After finishing training mode, tap coefficients are updated using the payload data. Fig. 4 shows BER curves for two types of channel equalizer in the environment of time varying channel during the payload data period. In time invariant channel, both of equalizers are well operated, however zero forcing method are not estimated time varying channel.

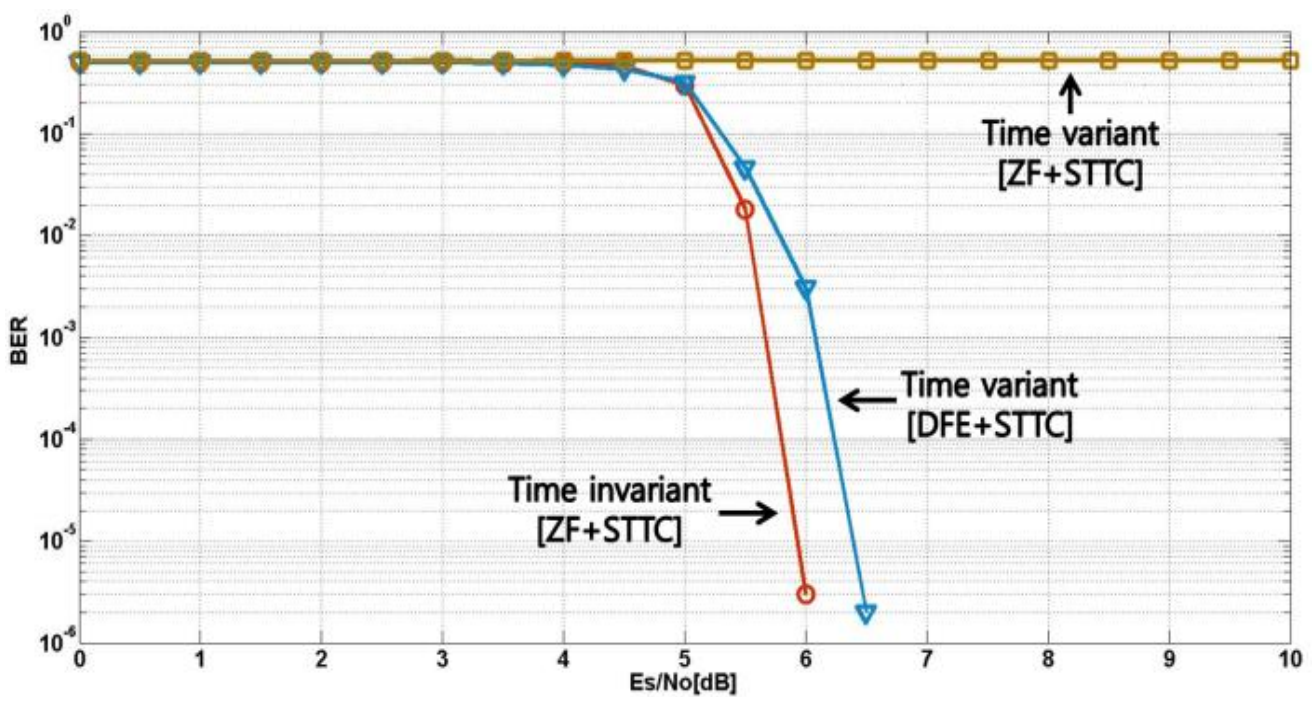

Fig. 4 The performance comparison of ZF and LMS-DFE

Performance of iteration structure. As above mentioned, we decided STTC as outer coding method, and DFE method as channel equalizer. Fixed on outer codes and DFE equalizer, we simulated performance according to iteration structure. At the receiver sides as shown in Fig. 5, the turbo codes are employed as inner codes in the paper. As the same as SISO communication, we resort to powerful turbo equalization algorithms that iteratively exchange probabilistic information between inner decoder and outer decoder, thereby reducing the error rates significantly. There are two scenarios to make iterative structure. First, the outer loop structure (mark "2" in Fig. 5) which the extrinsic information of turbo decoder are input to the DFE equalizer. Second, the inner loop structure (mark "1" in Fig. 5) which the extrinsic information of turbo decoder are input to the BCJR decoder. As shown in Fig. 6, the inner loop structure has coding gains compare to outer loop one slightly.

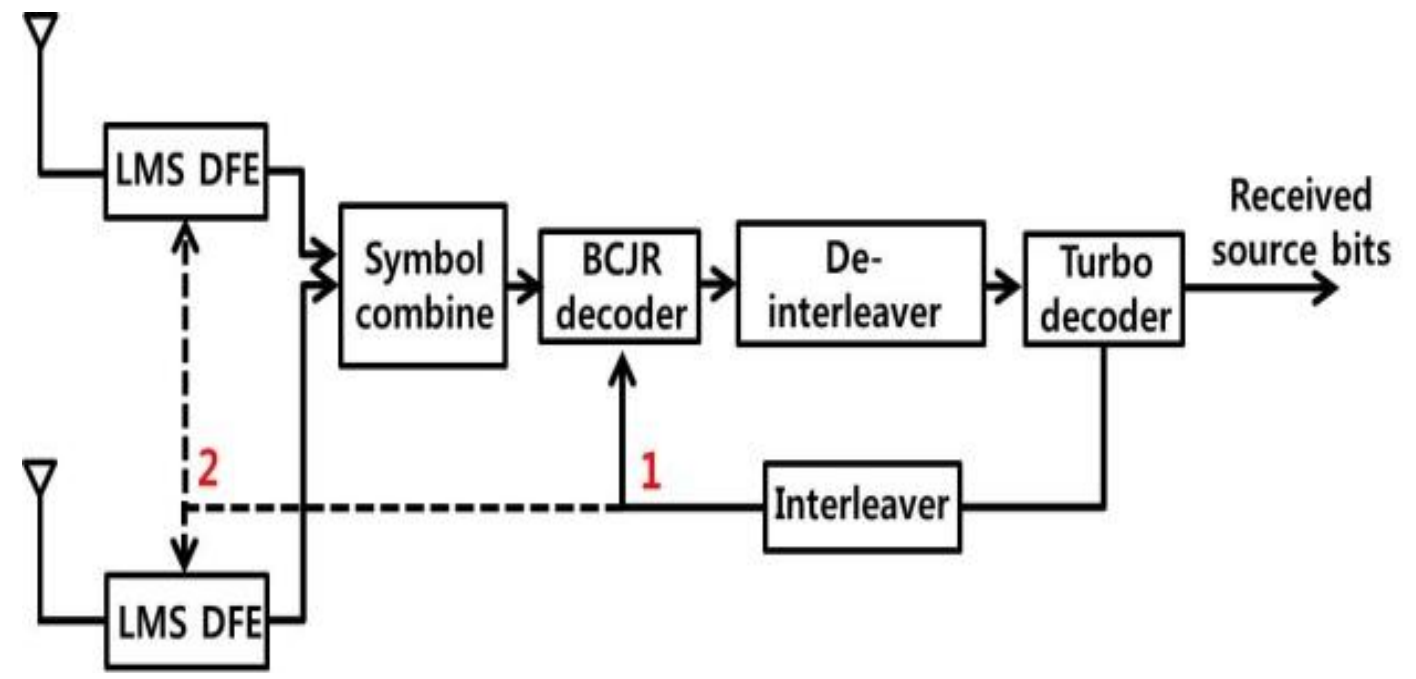

Fig. 5 The performance comparison of ZF and LMS-DFE 


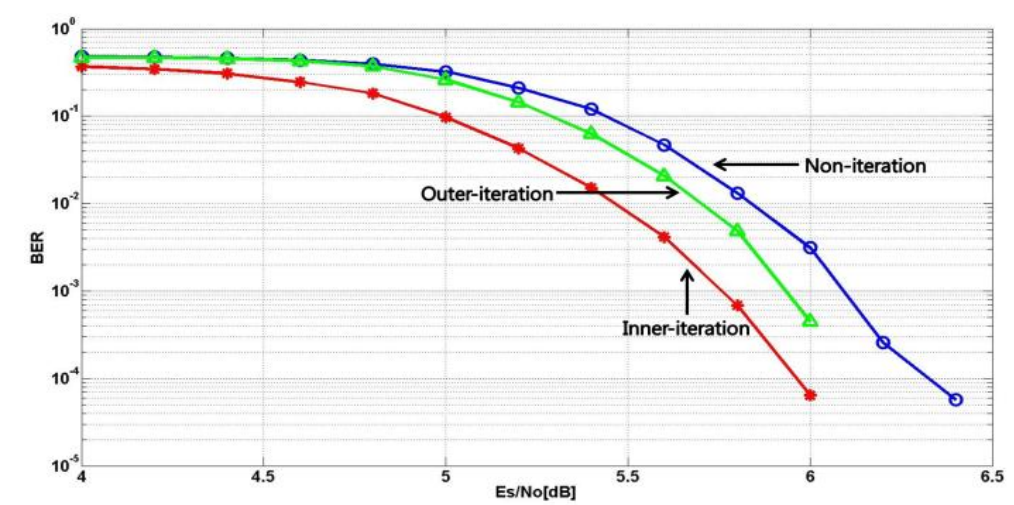

Fig. 6 The performance comparison of ZF and LMS-DFE

\section{Conclusions}

In this paper, we proposed receiver structure based on an iterative turbo equalization to cope with inter symbol interference and MIMO underwater communication channel. Iterative MIMO turbo equalizer consists of inner codes and outer codes. Through simulations by fixing the turbo codes as inner codes, we confirmed STTCs with BCJR algorithm are optimal outer codes, and DFE method is optimal for channel equalizer for MIMO underwater channel. Furthermore, we presented optimal iterative structure in order to improve the performance by increasing number of iterations.

\section{Acknowledgements}

This work was supported by Defense Acquisition Program Administration and Agency for Defense Development under the contract UD130007DD.

\section{References}

[1] M. Tuchler, R. Koetter, and A. Singer, Turbo Equalization : Principles and New Results, IEEE Trans. Communications, Vol.50, no.5, May (2002), pp.754-767

[2] R. Koetter, A. C. Singer, and M. Tuchler, Turbo equalization, IEEE Signal Process. Mag., Vol. 21, no. 1, Jan. (2004), pp. 67-80

[3] J. Ling, T. Yardibi, X. Su, H. He, and J. Li, Enhanced Channel Estimation and Symbol Detection for High Speed Multi-input Multi-output Underwater Acoustic Communications, J. Acoust. Soc. Am., 125, (2009) , pp.3067-3078

[4] H. C. Song, P. Roux, W. S. Hodgkiss, W. A. Kuperman, T. Akai, and M. Stevenson, Multiple-input-multiple-output coherent time reversal communications in a shallow-water acoustic channel, IEEE J. Ocean. Eng., Vol.31, Jan. (2006) , pp.170-187

[5] Subhadeep Roy, Tolga M. Duman, Vincent McDonald, and John G. Proakis High-Rate Communication for Underwater Acoustic Channels Using Multiple Transmitters and Space-Time Coding: Receiver Structures and Experimental Results, IEEE J. Oceanic Eng., Vol.32, (2007).

[6] L. R. Bahl, J. Cocke, F. Jelinek, and J. Raviv, Optimal decoding of linear codes for minimizing symbol error rate, IEEE Trans. Inf. Theory, Vol. IT-20, no. 2, Mar. (1974) , pp. 284-287

[7] G.D. Golden, G.J. Foschini, R.A. Valenzuela, and P.W. Wolniansky, Detection Algorithm and Initial Laboratory Results using V-BLAST Space-time Communication Architecture, Electron. Lett., Nov. (1998). 
[8] P. W. Wolniansky, G. J. Foschini, G. D. Golden, and R. A. Valenzuela, V-BLAST: An architecture for realizing very high data rates over the rich-scattering wireless channel, in Proceedings of the ISSSE, Pisa, Italy, (1998), pp. 295-300

[9] C. Carbonelli, S. Vedantam, and U. Mitra, Sparse channel estimation with zero tap detection, in IEEE International Conference on Communications, Paris, France, Vol. 6, (2004), pp. $3173-3177$ 